\title{
COUPLED NONPARAMETRIC SHAPE PRIORS FOR SEGMENTATION OF MULTIPLE BASAL GANGLIA STRUCTURES
}

\author{
Gokhan Uzunbas, Mujdat Cetin, Gozde Unal, and Aytul Ercil \\ Sabanci University \\ Faculty of Engineering and Natural Sciences \\ Tuzla, 34956 \\ Istanbul,Turkey
}

\begin{abstract}
This paper presents a new method for multiple structure segmentation, using a maximum a posteriori (MAP) estimation framework, based on prior shape densities involving nonparametric multivariate kernel density estimation of multiple shapes. Our method is motivated by the observation that neighboring or coupling structures in medical images generate configurations and co-dependencies which could potentially aid in segmentation if properly exploited. Our technique allows simultaneous segmentation of multiple objects, where highly contrasted, easy-to-segment structures can help improve the segmentation of weakly contrasted objects. We demonstrate the effectiveness of our method on both synthetic images and real magnetic resonance images (MRI) for segmentation of basal ganglia structures.
\end{abstract}

Index Terms - MRI, brain, Basal Ganglia, curve evolution, multi object image segmentation, shape priors, nonparametric shape density.

\section{INTRODUCTION}

Medical image segmentation has improved the diagnosis of many human diseases through more robust and accurate extraction of relevant tissues from medical image data. One of the main motivations for the segmentation of subcortical structures in the brain MR images is the analysis of chemicals in various basal ganglia (BG) structures for diagnosis of neurodegenerative diseases. The amount of chemical accumulation and shape deformations in subcortical structures are thought to provide important clues in early diagnosis. However, the information obtained from these images does not often provide enough contrast or clear boundary patterns of subcortical structures. Under such conditions, use of prior information based on shape is gaining increased attention in MR image segmentation algorithms.

Introducing shape priors into deformable models is not a novelty. There are numerous existing automatic segmentation methods that enforce constraints about the underlying shapes. In a typical active contour-based image segmentation, a curve length penalty term is often used for regularization, which makes the assumption that shorter curves are in general more likely than the longer ones [1]. However, in many applications, more structured prior information about the shapes is available. In [2] [3], principal component analysis (PCA) of the signed distance functions of training data is used to capture the variability of shapes. However, these techniques can

This work was partially supported by the European Commission under Grants FP6-2004-ACC-SSA-2 (SPICE), MIRG-CT-2006-041919 and MTKI-CT-2006-042717 be applied only when the shape variability is rather small. Secondly, these techniques can handle only unimodal, Gaussian-like shape densities. In particular, these methods cannot deal with multimodal shape densities, which involve multiple classes of shapes. As a solution to the limitation of PCA and unimodal Gaussian distribution models, techniques based on nonparametric shape densities learned from example training shapes have been proposed in [4], [5].

Although a nonparametric prior is very adequate to capture nonlinear shape variability, some challenging applications in medical image segmentation still require some additional information to be used. In many cases, objects to be segmented, have one or more neighboring structures, whose location and shape provide information about the local geometry that can aid in the delineation. In this fashion, the relative shape arrangements among these neighbors can be modeled based on statistical information from a training set. In [6], a joint prior based on a parametric shape model, is proposed to capture co-variations shared among the different shape classes, which improves the performance of segmentation. With a similar approach in a Bayesian framework, in [7], joint prior information of the multiple objects is used to capture the dependencies among the different shapes where multiple objects with clearer boundaries are used as reference objects to provide constraints in the segmentation of poorly contrasted objects. Another coupled shape prior model, which is based on the cumulative distribution function of shape features, is proposed in [8]. In [8], relative inter-object distances are defined as a shape feature to capture information about the interaction between multiple objects.

In this paper, having the inspiration of capturing co-variations and dependencies between different shapes, we propose a new method that extends nonparametric shape priors to the problem of coupled segmentation of multiple objects. We use multivariate density estimation to estimate unknown joint distribution of multiple shapes which effectively captures coupling between distinct shapes in a scene. This key property of our method allows segmenting multiple objects simultaneously where the new prior provides automatical, coupled constraints to be used in challenging image scenarios. Moreover, as compared to existing methods [6], [7] which are based on prior of multiple objects, our approach takes the advantage of using nonparametric density estimate, in order to capture non-linear shape variability.

\section{SEGMENTATION FRAMEWORK BASED ON COUPLED SHAPE PRIORS}

In a typical active contour model, the segmentation process involves an iterative algorithm for minimization of an energy functional. We 
define our energy (cost) functional in a MAP estimation framework as

$$
E(\mathbf{C})=-\log p(\operatorname{data} \mid \mathbf{C})-\log p_{\mathbf{C}}(\mathbf{C})
$$

where $\mathbf{C}$ is a set of evolving curves $\left\{C^{1}, \ldots, C^{m}\right\}$ that match the boundary of the shapes. The first term, $\log p($ data $\mid \mathbf{C})$, called the likelihood term, enforces some form of data fidelity; and $\log p_{\mathbf{C}}(\mathbf{C})$ is a joint prior density for the shapes involved. In particular, we choose the likelihood term as in [9], which is a common technique used in previous works such as [3], [5], [6], [7]. In this paper, we focus on building the coupled shape prior $p_{\mathbf{C}}(\mathbf{C})$.

\subsection{Coupled Prior Model of Multiple Objects}

Here the essential idea is to construct coupled nonparametric shape prior information $p_{\mathbf{C}}(\mathbf{C})$ for $m$ different class of objects. Let us assume we are given $N$ example training samples $\left\{\mathbf{C}_{1}, \ldots, \mathbf{C}_{N}\right\}$. Each "sample" consists of multiple shapes, each of which corresponds to one of $m$ objects, i.e $\mathbf{C}_{i}=\left\{C_{i}^{1}, \ldots C_{i}^{m}\right\}$. The pose variation in the training data is removed by an alignment operation, as in [3]. In this operation we calculate a set of similarity transformation parameters (translation, scaling and rotation) for each sample in the training set to align binary shapes with each other. We align each sample of the $m^{t h}$ object within its own members and we obtain $\left\{\tilde{\mathbf{C}_{1}}, \ldots, \tilde{\mathbf{C}_{N}}\right\}$. After alignment, any variability due to pose differences is removed and what remains is shape variation. Fig. 1 shows binary aligned shape representations for 2 objects (head of caudate and putamen) from 12 different slices.

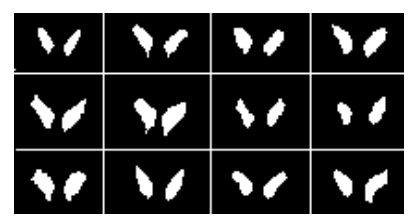

(a)

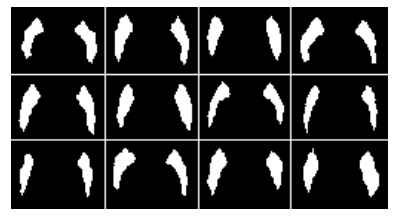

(b)
Fig. 1. Aligned Training Shapes for (a) head of caudate and (b) putamen extracted from multi-modality sequences.

To build a joint prior model for multiple objects, we choose level sets as the representation of shapes [10] and we use multivariate Parzen density estimation (see [11]) to estimate the unknown joint shape distribution. For the sake of simplicity of exposition and without loss of generality, let us take $m=2$, and then, define the joint kernel density estimate of two shapes as,

$$
p_{\tilde{C^{1}}, \tilde{C}^{2}}\left(\tilde{C^{1}}, \tilde{C}^{2}\right)=\frac{1}{N} \sum_{i=1}^{N} \prod_{k=1}^{m=2} k\left(d\left(\phi_{\tilde{C}^{k}}, \phi_{\tilde{C}_{i}^{k}}\right), \sigma_{k}\right)
$$

where $k\left(., \sigma_{k}\right)$ is a Gaussian kernel with standart deviation $\sigma_{k}$. In this equation, $\phi_{\tilde{C}^{k}}$ is the candidate signed distance function and $\phi_{\tilde{C}^{k}}$ is the signed distance function for the $i^{\text {th }}$ training shape of the $k^{t h}$ object. Note that, given a distance measure $d(.,$.$) , we can construct$ the kernel function for joint density estimation, by multiplying separate kernels $k\left(., \sigma_{k}\right)$ for each object. Our nonparametric shape prior in (2) can be used with a variety of distance metrics. In this work, we consider two specific metrics, namely the template metric and the $L_{2}$ distance $d_{L_{2}}$ between signed distance functions. For the kernel size $\sigma_{k}$, for the $k^{t h}$ object, we use maximum likelihood kernel size with leave-one-out [12].

As compared to existing single shape prior based approaches [3], [5] in this framework, we produce much more accurate joint shape densities in cases where there are shape dependencies between the multiple objects involved. This is a phenomenon we observe in basal ganglia structures.

\subsection{Gradient Flow for the Coupled Shape Prior}

In this part, we compute the gradient flow of the joint prior in equation (2) for the two curves which are represented implicitly by their corresponding signed distance functions. We adress how the gradient flow for the coupled shape prior is obtained with gradient descent optimization.

In differentiating the logarithm of the expession given in (2), we use shorthand notation, $k_{\sigma_{k}}$ for $k\left(d_{L_{2}}\left(\phi_{C^{k}}, \phi_{C_{i}^{k}}\right), \sigma_{k}\right)$. Note that $\phi_{\tilde{C^{k}}}$ is a function of time $\mathrm{t}$ and $\phi_{\tilde{C^{k}}}$ is a shorthand notation for the evolving level set function $\phi_{\tilde{C^{k}}}(t)$. Using these, we obtain

$$
\frac{\partial}{\partial t} \log p_{\tilde{C}^{1}, \tilde{C}^{2}}\left(\tilde{C^{1}}, \tilde{C}^{2}\right)=\frac{1}{N} \frac{\sum_{i=1}^{N}\left\{k_{\sigma_{1}}^{\prime} k_{\sigma_{2}}+k_{\sigma_{1}} k_{\sigma_{2}}^{\prime}\right\}}{p_{\tilde{C}^{1}, \tilde{C}^{2}}\left(\tilde{C}^{1}, \tilde{C}^{2}\right)}
$$

Then, we compute the gradient direction that increases most rapidly for each object curve. Using the $L_{2}$ distance in kernels, we find that the gradient directions for the curves $\tilde{C^{k}}$, where $k=1,2$, are

$$
\frac{\partial \phi_{\tilde{C^{k}}}}{\partial t}=\frac{1}{\sigma_{k}^{2}} \sum_{i=1}^{N} \lambda_{i}\left(\tilde{C^{1}}, \tilde{C}^{2}\right)\left(\phi_{\tilde{C}_{i}^{k}}(x)-\phi_{\tilde{C^{k}}}(x)\right)
$$

where $\lambda_{i}\left(\tilde{C^{1}}, \tilde{C}^{2}\right)=\frac{k\left(d_{L_{2}}\left(\phi_{\tilde{C}^{1}}, \phi_{\tilde{C}_{i}^{1}}\right), \sigma_{1}\right) k\left(d_{L_{2}}\left(\phi_{\tilde{C}^{2}}, \phi_{\tilde{C}_{i}^{2}}\right), \sigma_{2}\right)}{N p_{\tilde{C}^{1}}, \tilde{C}^{2}\left(\tilde{C}^{1}, \tilde{C}^{2}\right)}$, and $\sum_{i=1}^{N} \lambda_{i}\left(\tilde{C}^{1}, \tilde{C}^{2}\right)=1$. The final expression (4), evolves the curves toward shapes at the local maximum of the coupled shape prior of two objects. Note that, training shapes that are closer to the evolving curve get more weight. Furthermore, the weighting function $\lambda_{i}$ depends on each curve in exactly the same way. In particular, due to the coupled nature of this weight, given a pair $\left(\tilde{C}_{1}, \tilde{C}_{2}\right)$ in the evolution process training shape pairs in which the second training shape is closer to $\tilde{C}_{2}$ get relatively more weight in the evolution of the first curve as well. This shows one aspect of the coupled nature of our shape-based segmentation aproach.

\subsection{Iterative Segmentation Algorithm}

Given an arbitrary test image, the objects to be segmented in the scene are not necessarily aligned with the shapes in our training set. Due to the pose differences, we relate the density estimates of candidate curves, $p_{\mathbf{C}}(\mathbf{C})$ with density estimates of aligned curves, $p_{\tilde{\mathbf{C}}}(\tilde{\mathbf{C}})$. In order to do that, describing each candidate curve by its shape and pose, we interpret $\tilde{\mathbf{C}}$ as the shape of $\mathbf{C}$ after being transformed by $T$ :

$$
\tilde{\mathbf{C}}=T[\mathbf{p}] \mathbf{C}
$$

where $T$ is a similarity transformation (translation, scaling and rotation) with parameters $\mathbf{p}=p^{1}, \ldots, p^{m}$ for each object and $m$ is the number of objects. Then during the evolution process, we align each candidate curve with its training shapes. For a review of the pose update algorithm we refer the reader to [5]. We can summarize the 
principal steps of our segmentation algorithm as follows:

1. Evolve $\mathbf{C}=C^{1}, \ldots, C^{m}$ until iteration time $t_{o}$ without shape prior

2. At $t=t_{0}$, for all $m$, compute the pose $\mathbf{p}=p^{1}, \ldots, p^{m}$

3. while $\left(t_{0}<\right.$ iterations $<$ maxIteration $)$

a ) fix $\mathbf{p}$, and for all $m$

- compute $\tilde{\mathbf{C}}=T[\mathbf{p}] \mathbf{C}$ using (Eqn. (5))

- compute $\frac{\partial \phi_{\tilde{C}}}{\partial t}$ for $\log p_{\mathbf{C}}(\mathbf{C})$ using (Eqn. (4))

- relate $\mathbf{C}=T^{-1}[\mathbf{p}] \tilde{\mathbf{C}}$

b ) Update $\mathbf{C}$ using both ${ }^{1}$ data and shape force

c ) fix $\mathbf{C}$ and update $\mathbf{p}$

\section{EXPERIMENTAL RESULTS}

In this section we show segmentation results for the head of caudate and the putamen which are two subcortical structures of the brain. We first consider 2D synthetic images created by adding noise over binary images representing the brain structures in Fig.1. These images are obtained through manual segmentation of true MR images. In training the set, we use 11 of these slices (with size $512 \times 448$ ) from four different patients and we perform segmentation on images which are not in the training set. We work with high contrasted caudate and low contrasted, occluded putamen to focus on the coupling effect of using the joint shape prior. We compare our results on putamen with those of [5], which uses independent priors for the two structures. Segmentation results with single prior and coupled prior for both structures are shown in Fig.2. The results for our method on putamen, visually show better segmentation than the results obtained by using a single prior.

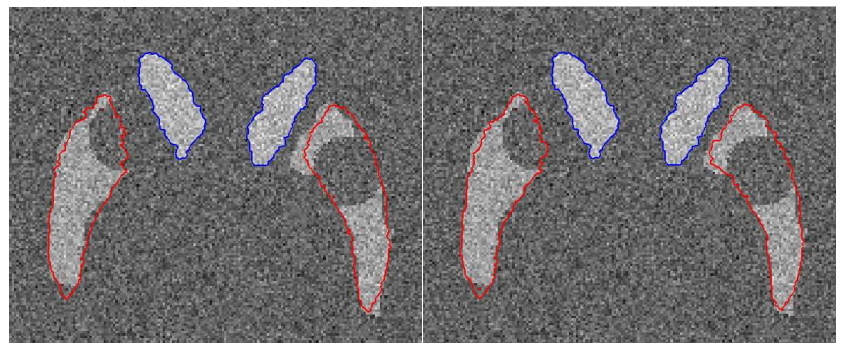

(a)

(b)

Fig. 2. Segmentation of the head of caudate (blue curve) and occluded putamen (red curve) (a) with single prior information and, (b) coupled prior information.

We also show quantitative comparison of the two methods by computing several performance measures such as false positive rate:

$$
F P R=\frac{F P}{F P+T N}
$$

false negative rate:

$$
F N R=\frac{F N}{F N+T P}
$$

\begin{tabular}{|l|c|c|c|}
\hline \multirow{2}{*}{ mean $\pm \sigma$} & \multicolumn{3}{|c|}{ Putamen } \\
\cline { 2 - 4 } & FPR (\%) & FNR (\%) & 1 - D.C. (\%) \\
\hline Single Prior & $0.76 \pm 2$ & $13.5 \pm 5.3$ & $16.8 \pm 5.9$ \\
\hline Coupled Prior & $0.70 \pm 2$ & $13.1 \pm 5.0$ & $13.8 \pm 4.2$ \\
\hline
\end{tabular}

Table 1. Average performance results on 12 synthetic images.

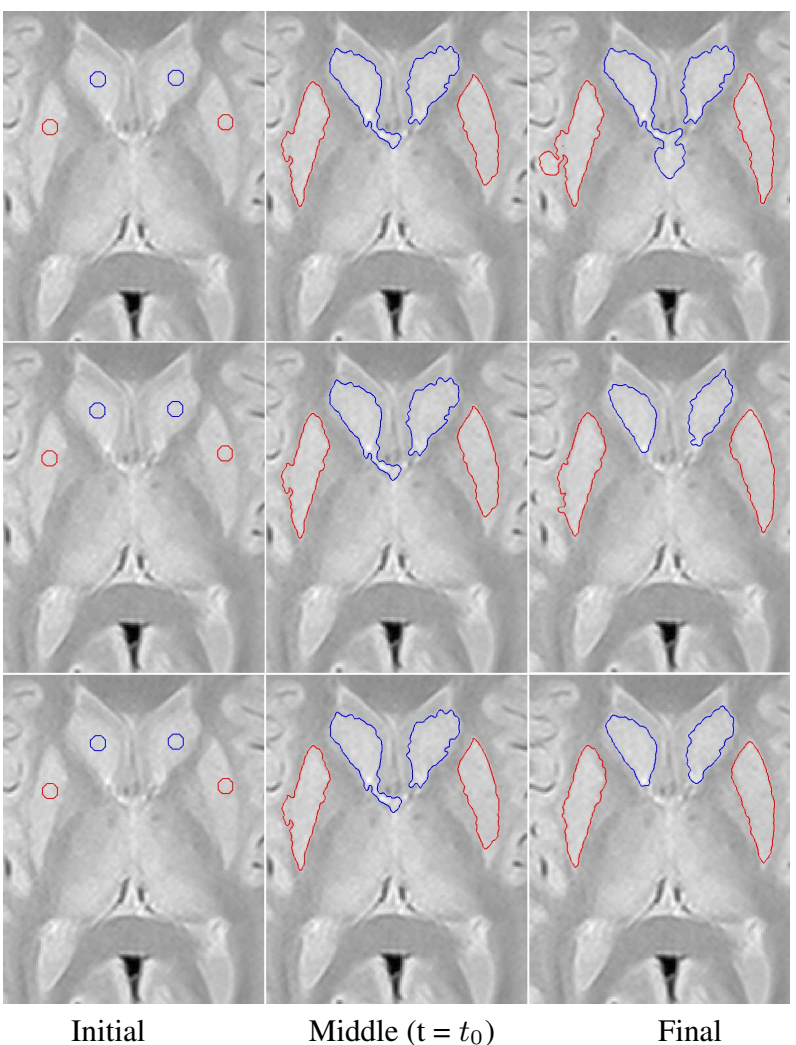

Fig. 3. Three steps in the segmentation of head of caudate and putamen in an MR slice with no prior (top row), with single prior (middle row) and with coupled prior information (bottom row).

Dice Coefficient (DC) (see [13]):

$$
D C=\frac{2 T P}{F P+F N+2 T P}
$$

where FP is false positive pixels inside the segmenting curve but out of the true shape, and FN is false negative pixels inside the true shape but out of the segmenting curve. TN is true negative pixels out of true shape and segmenting curve. TP is true positive pixels inside both true shape and segmenting curve. We present average values over 12 tests for 12 different images, in Table. 1.

We then consider Proton Density (PD) sequences of real MR data sets with 2 subcortical structures of interest, head of caudate and putamen. We use a training set of 11 pairs of shapes. Fig. 3 top row shows initial, middle and final steps of the segmentation using only gray level information as proposed in [9], where the head of caudate and left putamen cannot be segmented well, due to low contrast. The middle row in the Fig. 3 shows the result of using

\footnotetext{
${ }^{1}$ Data force can be obtained by computing the gradient flow of the first term in Eqn. (1)
} 
independent shape prior for each structure [5]. In this case, due to the leakage in putamen and mismatch in the right part of caudate, the result is not satisfactory. Fig. 3 bottom row shows the results of our coupled shape prior model, where the coupling between putamen and caudate improves the results.

In Table. 2, it can be observed that our method achieves a much lower FPR for putamen and FNR for caudate than the technique in [5]. According to (1 - DC) measure, our method performs better than the other methods for both objects.

\begin{tabular}{|l|c|c|c|c|c|c|}
\hline \multirow{2}{*}{$\%, \%, \%$} & \multicolumn{3}{|c|}{ Putamen } & \multicolumn{3}{c|}{ Head of Caudate } \\
\cline { 2 - 7 } & FPR & FNR & 1-D.C. & FPR & FNR & 1-D.C \\
\hline Without Prior & 1.10 & 5 & 12 & 4.2 & 8 & 22 \\
\hline Single Prior & 1.08 & 4 & 13 & 0.29 & 20 & 17 \\
\hline Coupled Prior & 0.88 & 4 & 8 & 0.35 & 14 & 13 \\
\hline
\end{tabular}

Table 2. Performance results on the real MR data set.

\section{CONCLUSIONS}

In this paper, we proposed an approach for introducing coupled shape prior into the segmentation process. We use multivariate Parzen density estimation to estimate the unknown joint distribution of multiple shapes. We introduce a new mechanism which automatically captures the coupling between distinct shapes in a scene. We evolve multiple curves in parallel with the influence of joint prior to segment poorly contrasted difficult shapes.

Our preliminary experiments demonstrated that our method has much promise for multi-object segmentation in medical imaging applications. This current work does not include any pose constraints on relative positions of coupled shapes. Moreover, the data term that we use, can be incorporated with some statistical priors based on intensity characteristics of the tissues. We are currently working on incorporating these approaches to our pricipal approach in order to improve our segmentation results in more challenging scenarios. We are working on segmentation of other subcortical structures, as well.

\section{ACKNOWLEDGMENT}

The authors would like to thank Junmo Kim for providing his code for nonparametric shape prior. The authors would also like to thank Dr. Kutlay Karaman, radiologist at Anadolu Medical Centre, for very helpful conversations and for providing the MR data.

\section{REFERENCES}

[1] Mumford D. and Shah J., "Boundary detection by minimizing functionals," IEEE Conferance Computer Vision Pattern Recognition, 1985.

[2] M. Leventon, E. Grimson, and O. Faugeras, "Statistical shape influence in geodesic active contours," IEEE Conferance Computer Vision Pattern Recognition, 2000, pp. 316-323.

[3] A. Yezzi, A. Tsai, W. Wells, C. Tempany, D. Tucker, A. Fan, W.E. Grimson, and A. Willsky, "A shape-based approach to the segmentation of medical imagery using level sets," IEEE Transactions Medical Imaging, vol. 22, pp. 137-154, 2003.

[4] D. Cremers, S.J. Osher, and S. Soatto, "Kernel density estimation and intrinsic alignment for shape priors in level set segmentation," Internat. J. Comp. Vision, vol. 69, no. 3, pp. 335-351, 2006.

[5] J. Kim, M. Cetin, and A.S. Willsky, "Nonparametric shape priors for active contour-based image segmentation," Signal Processing, vol. 87, pp. 3021 - 3044, 2007.

[6] A. Tsai, W. Wells, C. Tempany, W.E. Grimson, and A. Willsky, "Mutual information in coupled multi-shape model for medical image segmentation," Medical Image Analysis, vol. 8, pp. 429445, 2004.

[7] J. Yang and J. Duncan, "Joint prior models of neighboring objects for 3-d image segmentation," IEEE Conferance Computer Vision Pattern Recognition, Washington, DC, 2004.

[8] A. Litvin and W.C. Karl, "Coupled shape distribution-based segmentation of multiple objects," Information Processing in Medical Imaging, 2005, pp. 345-356.

[9] T. Chan and L. Vese, "Active contours without edges," IEEE Transactions Image Processing., vol. 2, pp. 266-277, 2001.

[10] S. Osher and R. Fedkiw, "Level set methods and dynamic implicit surfaces," Springer, Berlin, 2003.

[11] D. Erdogmus, R. Jenssen, Y.N. Rao, and J.C. Principe, "Gaussianization: An efficient multivariate density estimation technique for statistical signal processing," Journal of VLSI Signal Processing, vol. 45, pp. 67-83, 2006.

[12] B.W. Silverman, "Density estimation for statistics and data analysis," Chapman Hall, London, 1986.

[13] L. Dice, "Measures of the amount of ecologic association between species," Ecology, vol. 26, pp. 297-302, 1945. 